\title{
CIÊNCIA'NATURA
}

\section{Influência da radiação de onda longa incidente no fechamento do balanço de radiação no modelo SIB2: Estimativas para pastagem no bioma Pampa}

Influence of long-wave radiation incident on radiation balance closure in model SIB2: Estimates for pasture in the Pampa biome

Daniele Morgenstern Aimi ${ }^{1}$, Maria Eduarda Oliveira ${ }^{1}$, Tamires Zímmer ${ }^{1}$, Gisele Cristina Rubert ${ }^{2}$, Vanessa de Arruda Souza ${ }^{2}$ e Debora Regina Roberti ${ }^{3}$

${ }^{1}$ Curso de Pós Graduação em Física, UFSM

${ }^{2}$ Curso de Pós Graduação em Meteorologia, UFSM

${ }^{3}$ Departamento de Física, UFSM

\section{Resumo}

O saldo de radiação representa os ganhos e perdas de energia na superficie, podendo ser estimado através da soma das quatro componentes da radiação em superficie (radiação solar incidente e refletida, radiação emitida pela superficie e emitida pela atmosfera). O modelo SiB2 representa ecossistemas e fornece estimativas das trocas de energia em superficie. Neste trabalho, foi testado cinco diferentes equações de estimativa de onda longa incidente para verificar a influência desta componente no fechamento do balanço de radiação para o modelo SiB2. Os resultados obtidos mostram a subestimativa no saldo de radiação do modelo SiB2 quando comparado aos dados experimentais. Deste modo, infere-se a necessidade de calibração das equações de radiação de onda longa incidente para a utilização nos modelos para simulações locais.

Palavras-chave: Balanço de radiação. Radiação de onda longa incidente. SiB2

\section{Abstract}

The balance of radiation represents the energy gains and losses on the surface, and can be estimated by summing the four components of surface radiation (incident and reflected solar radiation, radiation emitted by the surface and emitted by the atmosphere). The SiB2 model represents ecosystems and provides estimates of surface energy changes. In this work, five different incident long wave estimation equations were tested to verify the influence of this component on the closure of the radiation balance for the SiB2 model. The results show the underestimation of the radiation balance of the SiB2 model when compared to the experimental data. Thus, it is inferred the need for calibration of incident long-wave radiation equations for use in models for local simulations.

Keywords: Radiation balance. Incident long wave radiation. SiB2 


\section{Introdução}

O saldo de radiação representa a energia utilizada para os processos de trocas entre a superfície, a atmosfera e o solo ou ainda aos processos físicos e biológicos que ocorrem no sistema superfície-atmosfera (OKE, 2002). O saldo de radiação é composto por quatro componentes de radiação: a radiação solar incidente $(\mathrm{K} \downarrow)$ e refletida $(\mathrm{K} \uparrow)$ e a radiação emitida pela superfície (L $\uparrow)$ e emitida pela atmosfera para a superfície $(\mathrm{L} \downarrow)$. Este saldo é particionado em diferentes processos que utilizam a energia na forma de calor para ocorrerem na natureza, como: condução, convecção, evaporação, condensação, transpiração e fotossíntese.

A radiação de onda longa proveniente da atmosfera é difícil de ser estimada devido a complexidade existente na região da troposfera como, por exemplo, ventos, variações na temperatura, na umidade, nos fluxos de massa e momentum, etc. (PIMENTEL, 2010). A maioria das estações meteorológicas de superfície raramente incluem sensores de radiação de ondas longas, de modo que as medições geralmente não estão disponíveis para um determinado local e período de interesse. Embora os fluxos radiativos de superfície possam ser calculados com razoável precisão utilizando modelos complexos de transferência radiativa (EDWARDS E SLINGO, 1996; POPE et al., 2000), estes requerem medições detalhadas da coluna de ar acima de um local, incluindo propriedades de nuvem e perfis verticais de temperatura, vapor de água, gases e aerossóis. Como essas medições raramente estão disponíveis, a radiação de longa é comumente estimada a partir de equações que usam observações meteorológicas disponíveis (DUARTE et al., 2006).

O modelo SiB2 (Simple Biosphere Model versão 2) foi desenvolvido para representar os ecossistemas e fornecer estimativas das trocas de energia, massa e momento entre a superfície e a atmosfera terrestre (SELLERS et al., 1996). Estas trocas são dependentes da correta prescrição dos parâmetros que representam o solo, da vegetação e das condições atmosféricas observadas no período de simulação.

O bioma Pampa abrange uma área de aproximadamente $176.496 \mathrm{~km}^{2}$ no extremo sul do Brasil (IBGE, 2011), correspondendo a $63 \%$ do território do estado do Rio grande do Sul e a 2,07\% do território brasileiro. As paisagens naturais do Pampa se caracterizam pelo predomínio dos campos nativos, mas há também a presença de matas ciliares, matas de encosta, matas de pau-ferro, formações arbustivas, butiazais, banhados, afloramentos rochosos entre outros (BORBA, 2007).

O objetivo deste trabalho é testar diferentes metodologias para o cálculo de um dos parâmetros do modelo SiB2, a radiação de onda longa incidente $(\mathrm{L} \downarrow)$, fazendo uso de equações descritas na literatura e analisar a influência desta no fechamento do balanço de radiação comparando com resultados obtidos experimentalmente sobre a região do Pampa no Sul do Brasil.

\section{Materiais e métodos}

\subsection{Descrição do sítio}

O sítio experimental de Santa Maria-RS localiza-se em uma área de campo nativo no bioma Pampa, dentro do campus da Universidade Federal de Santa Maria (UFSM) (Latitude: -29,7241 ${ }^{\circ}$; Longitude: $-53,7597^{\circ}$; Altitude: $88 \mathrm{~m}$ ). Neste local, está uma torre micrometeorológica com sensores que medem as variáveis ambientais utilizadas para inicializar o SiB2, Tabela1.

Tabela 1- Instrumentação do Sítio Experimental de Santa Maria no período de estudo.

\begin{tabular}{|c|c|c|c|}
\hline Instrumento & Variável medida & Modelo/Marca & Altura (m) \\
\hline Anemômetro Sônico & $\mathrm{u}, \mathrm{v}, \mathrm{w}$, temperatura do sônico & Wind Master Pro/Gill Instruments & 3 \\
\hline Saldo radiômetro & saldo de radiação & CNR4/ Kipp\&Zonen & 3 \\
\hline Pluviômetro & Precipitação & TR525USW/Texas electronics, Inc. & 6 \\
\hline Termohigrômetro & Temperatura e umidade do ar & HMP155/Vaisala & 3 \\
\hline
\end{tabular}

\subsection{Simple Biosphere Model (SiB2)}

O modelo SiB2, descrito por Sellers et al. (1996) é um modelo usado para calcular a transferência de energia, massa e momentum entre a atmosfera e a superfície de acordo com a com a cobertura vegetal.

Estas trocas são dependentes dos parâmetros que representam o solo e a vegetação e das condições atmosféricas observadas no período de simulação. Para a realização das simulações com o modelo SiB2 são necessários os parâmetros que representem o solo, a vegetação (dados da literatura) e as condições atmosféricas da área de estudo (sítio experimental) em escala horária.

\subsection{Saldo de Radiação}

O saldo de radiação (Rn) em superfície, é a soma da radiação de onda curta incidente $(K \downarrow)$ e refletida $(K \uparrow)$ e a radiação de onda longa emitida pela superfície $(\mathrm{L} \uparrow)$ e pela atmosfera $(\mathrm{L} \downarrow)$. Resultando na seguinte expressão: 


$$
\mathrm{R}_{\mathrm{n}}=\mathrm{K} \downarrow+\mathrm{K} \uparrow+\mathrm{L} \downarrow+\mathrm{L} \uparrow
$$

$\mathrm{O}$ modelo SiB2 calcula as três componentes do saldo de radiação, $\mathrm{K} \uparrow, \mathrm{L} \uparrow$ e $\mathrm{L} \downarrow$, sendo a radiação de onda curta incidente na superfície fornecida como dado de entrada para inicialização do modelo.

As componentes da radiação de onda longa, L $\uparrow$ e $L \downarrow$, são calculadas segundo a lei de Stefan- Boltzamnn. Neste trabalho, foram selecionadas cinco equações de $\mathrm{L} \downarrow$ mais utilizadas na literatura (Tabela 2), a fim de se avaliar quais destas representam mais significativamente o balanço de radiação na área de estudo, utilizando o modelo SiB2. Essas equações foram inseridas individualmente e para cada uma o modelo gerou um conjunto de dados os quais foram comparados com os dados experimentais. Foi então, estimado o balanço de radiação para cada uma das simulações realizadas.

$$
\left(0,79+0,17 * \exp \llbracket\left[\left(-\mathrm{e}^{*} 0,96\right)\right)^{*} \llbracket \sigma \mathrm{T} \rrbracket \wedge 4 \rrbracket\right.
$$

Tabela 2 - Equações de $\mathrm{L} \downarrow$ descritas na literatura, $\sigma=5.67 \times 10^{-8} \mathrm{~W} \mathrm{~m}^{-2} \mathrm{~K}^{-4}$.

\begin{tabular}{c|c|c}
\hline Teste & Referência & Equação de L $\downarrow$ \\
\hline L1 & Garrat (1992) & $(0,79+0,17 * \exp (-\mathrm{e} * 0,96)) * \sigma \mathrm{T} 4$ \\
\hline L2 & Niemalä 2001 & $(0,72+(0,009(\mathrm{e}-2))) * \sigma \mathrm{T} 4$ \\
\hline L3 & Swinbank (1963) & $(9,2 \times 10-6 * \mathrm{~T} 2)^{*} \sigma \mathrm{T} 4$ \\
\hline L4 & Idso e Jackson (1969) & $(1-0,261 * \exp (-0,00077(\mathrm{~T}-273,13) 2) * \sigma \mathrm{T} 4$ \\
\hline L5 & Brutsaert (1975) & $(1,7223(\mathrm{e} / \mathrm{T}) 1 / 7)) \sigma \mathrm{T} 4$ \\
\hline
\end{tabular}

No modelo SiB2, a radiação de onda longa refletida é calculada segundo a equação:

$$
\mathrm{L} \uparrow=\left(v c *\left(1-\mathrm{T}_{\mathrm{k}}\right) * \sigma \mathrm{T}_{\mathrm{c}}^{4}\right)+\left(\left(1-v c^{*}\left(1-\mathrm{T}_{\mathrm{k}}\right)\right) * \sigma \mathrm{T}_{\mathrm{g}}{ }^{4}\right)
$$

Onde $v c$ é a fração verde da vegetação, $\mathrm{T}_{c}$ é a temperatura do dossel, $T_{g} \mathrm{~T}_{\mathrm{g}}$ é a temperatura da superfície e $\mathrm{T}_{\mathrm{k}}$ é a radiação térmica do dossel.

A radiação de onda curta refletida é calculada pela seguinte expressão:

$$
\mathrm{K} \uparrow=\mathrm{alb} * \mathrm{~K} \downarrow
$$

em que, alb é o albedo da superfície e estimado pelo modelo SiB2.

\subsection{Dados Experimentais}

Foram utilizados 36 meses de dados, entre os anos de 2014 a 2016, obtidos do sítio experimental de Santa Maria, localizado no campus da Universidade Federal de Santa Maria (Latitude: -29,7241 ${ }^{\circ}$; Longitude: -53,7597 ; Altitude: $88 \mathrm{~m}$ ).

Os dados experimentais são obtidos em alta frequência (10hz) e depois processados em médias de 60 minutos. Devido a falhas na armazenagem e quedas de energia foram utilizadas técnicas de preenchimento de falhas para os períodos com falta de dados. Para isto, utilizou-se dados da estação meteorológica automática do Instituto Nacional de Meteorologia (INMET) a qual se encontra a $4 \mathrm{~km}$ de distância da torre micrometeorológica. O preenchimento foi obtido através da regressão linear das seguintes variáveis: pressão atmosférica, radiação de onda curta incidente, umidade relativa do ar, intensidade do vento e temperatura do ar.

\section{Resultados e discussões}

O modelo SiB2 utiliza $\mathrm{K} \downarrow$ como dado de entrada e estima as demais componentes do balanço de radiação. Na Figura 1(a, b, c, d, e), é apresentada a dispersão entre os dados do saldo de radiação experimentais e simulados pelo modelo SiB2 com diferentes equações de radiação de onda longa incidente para todo período de dados. Nestas figuras também são apresentadas os índices estatísticos. Observa-se que o dado simulado pelo $\mathrm{SiB} 2$ subestima os dados experimentais para todo o período de estudo. Analisando os coeficientes estatísticos nota-se que os melhores resultados foram obtidos para as equações L3 e L4 
(Tabela 2). Isto pode estar relacionado ao fato de ambas utilizarem em sua formulação apenas a temperatura do ar. A equação L5 apresentou o menor R2 e maior RMSE .

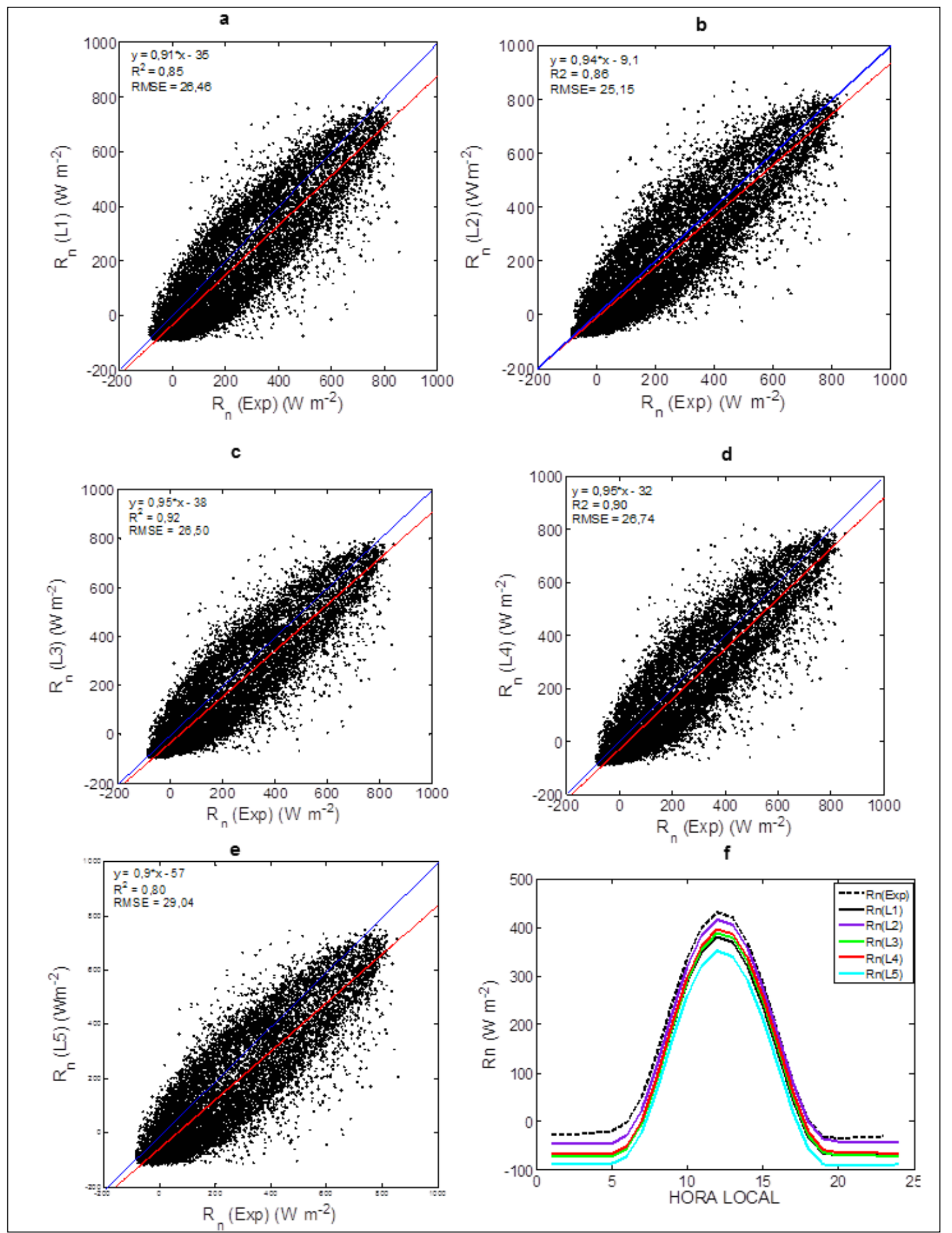

Figura 1- Dispersão entre dados experimentais e simulados com diferentes equações de radiação de onda longa incidente pelo modelo SiB2 no bioma Pampa:figura1 (a) L1; figura1 (b) L2; figura1 (c) L3; figura1 (d) L4; figura1 (e) L5 e figura1 (f) ciclo diário médio do saldo de radiação. Linhas coloridas continuas representam os resultado simulados pelo modelo SiB2 e a linha tracejada preta representa os dados experimentais. 
Na Figura 1 (f) são mostrados os ciclos diários médios do saldo de radiação para as diferentes equações simuladas. Observa-se que os resultados simulados pelo modelo $\mathrm{SiB} 2$ subestimaram os valores experimentais tanto para o período diurno quanto o noturno. No período de máxima insolação, aproximadamente as $12 \mathrm{~h}$ (hora local), ocorre a maior diferença entre dados experimentais e calculados pelo SIB2, cerca de $22 \%$ para L5 e a menor diferença entre os testes realizados foi o que utiliza a equação de Niemalä (2001), com uma diferença de 3,6\%.

\section{Conclusões}

As equações de radiação de onda longa incidente podem influenciar no fechamento do balanço de radiação no modelo SiB2. Dentre as cinco equações utilizadas neste trabalho, as que levam apenas em consideração a temperatura do ar apresentam as maiores subestimativas quando comparadas aos dados experimentais. Assim, os resultados obtidos neste trabalho sugerem a necessidade de calibrar as equações para dados locais.

\section{Agradecimentos}

PPGFísica - UFSM, CAPES, CNPQ e FAPERGS.

\section{Referências}

BORBA, M. F. S. Denominações de origem e o desenvolvimento regional: o exemplo do pampa. Embrapa Pecuária Sul-Documentos (INFOTECA-E), 2007.

BRUTSAERT, Wilfried. On a derivable formula for long-wave radiation from clear skies. Water Resources Research, v. 11, n. 5, p. 742-744, 1975.

DUARTE, H. F.; DIAS, N. L.; MAGGIOTTO, S. R.. Assessing daytime downward longwave radiation estimates for clear and cloudy skies in Southern Brazil. Agricultural and forest meteorology, v. 139, n. 3, p. 171-181, 2006.

EDWARDS, J. M.; SLINGO, A. Studies with a flexible new radiation code. I: Choosing a configuration for a largescale model. Quarterly Journal of the Royal Meteorological Society, v. 122, n. 531, p. 689-719, 1996.

GARRATT, J. R. Extreme maximum land surface temperatures. Journal of applied meteorology, v. 31, n. 9, p. 1096-1105, 1992.

IBGE. Censo Demográfico 2000 - Características Gerais da População. Resultados da Amostra. IBGE, 2003. Disponível em <http://www.ibge.gov.br/ home/estatistica/ populacao/cnso2000/default_populacao.shtm>. Acessado em 12 de setembro de 2017.

IDSO, Sherwood B.; JACKSON, Ray D. Thermal radiation from the atmosphere. Journal of Geophysical Research, v. 74, n. 23, p. 5397-5403, 1969.

NIEMELÄ, S.; RÄISÄNEN, P.; SAVIJÄRVI, H. Comparison of surface radiative flux parameterizations: Part I: Longwave radiation. Atmospheric Research, v. 58, n. 1, p. 1-18,

OKE, T.. Boundary Layer Climates, 2o edn. Taylor \& Francis e-Library, 2002.

PIMENTEL, R. M. M. 2010. Mudanças climáticas, relações hídricas e propriedades ópticas foliares. In: Mudanças Climáticas e Impactos Ambientais, Editora Universitária da UFPE, 169-177pp. 2001.

SELLERS, P. J. et al. A revised land surface parameterization (SiB2) for atmospheric GCMs. Part I: Model formulation. Journal of climate, v. 9, n. 4, p. 676-705, 1996.

SWINBANK, W. CQJR. Long-wave radiation from clear skies. Quarterly Journal of the Royal Meteorological Society, v. 89, n. 381, p. 339-348, 1963. 
Daniele Morgenstern Aimi

Universidade Federal de Santa Maria, Campus de Santa Maria, Santa Maria, RS, Brasil E-mail: danielefm@gmail.com

Maria Eduarda Oliveira

Universidade Federal de Santa Maria, Campus de Santa Maria, Santa Maria, RS, Brasil E-mail: mariaeduardafisica@gmail.com

Tamíres Zimmer

Universidade Federal de Santa Maria, Campus de Santa Maria, Santa Maria, RS, Brasil

E-mail: tz.tamireszimmer@gmail.com

Gisele Cristina Rubert

Universidade Federal de Santa Maria, Campus de Santa Maria, Santa Maria, RS, Brasil E-mail: girubert@gmail.com

Vanessa de Arruda Souza

Universidade Federal de Santa Maria, Campus de Santa Maria, Santa Maria, RS, Brasil E-mail: v.arruda.s@gmail.com

\section{Debora Regina Roberti}

Universidade Federal de Santa Maria, Campus de Santa Maria, Santa Maria, RS, Brasil E-mail: debora@ufsm.com 